This is the peer reviewed version of the following article: Chiong, Winston. "Therapeutic misconceptions and moral conflict in clinical research." Annals of neurology 67.4 (2010): 419-421., which has been published in final form at https://doi.org/10.1002/ana.22003. This article may be used for non-commercial purposes in accordance with Wiley Terms and Conditions for Use of Self-Archived Versions.

\title{
Therapeutic misconceptions and moral conflict in clinical research
}

\author{
Winston Chiong, MD PhD
}

Consider a physician whose patient meets inclusion criteria for a randomized clinical trial centered at a nearby institution, where the clinical trial has been undertaken to resolve a controversy regarding which of 2 treatment plans is more efficacious in this patient's condition. The physician must then choose whether to treat the patient "empirically" by prescribing the treatment that she judges most likely to benefit the patient, or to recommend enrollment in the clinical trial where the patient will be randomized to 1 of 2 treatment arms. Intuitively, this physician should consider the interests of 2 different parties: her present patient, who could be randomized to a treatment that she believes to be less likely to benefit him; and future patients with similar conditions, who stand to benefit from medical knowledge gained by testing interventions in a controlled fashion.

Clinical researchers and bioethicists have long recognized a potential ethical conflict in clinical research, between the clinical aim of benefiting the individual patient and the scientific aim of producing generalizable data to guide the care of future patients. (The equipoise requirement represents an established, but I think ultimately unsatisfactory, attempt at resolving this conflict. ${ }^{1,2,3}$ ) However, in their article in this issue of Annals of Neurology, Patrick Lyden and colleagues acknowledge no conflict between these aims. They claim that clinical trial enrollment is superior to empiric treatment both from the perspective of the individual patient and from the perspective of future patients, and that empiric treatment would therefore be unethical. They go on to propose a blanket approach to clinical decision making in medical emergencies (although most of their arguments are applicable to clinical practice quite generally) in which clinical trial enrollment is prioritized over empiric treatment. ${ }^{4}$

Let us grant that enrolling this patient in a clinical trial would promote the interests of future patients. I wish to focus instead on Lyden and colleagues' repeated assertion that trial enrollment is also best for the individual patient who is enrolled in the study. The grounds for this assertion are weak. First, they claim that "Most available data suggest that patients enrolled in trials may benefit, regardless of the treatment group into which they are randomized." Much appears to depend on what these authors mean by "most," "suggest," and "may"; the 2 sources cited are a 2001 meta-analysis of 21 studies that concludes that there may be such an effect but the evidence is poor ${ }^{5}$ and a 2008 meta-analysis of 85 studies (including many of the same studies, and excluding several

\footnotetext{
${ }^{1}$ Hill AB. Medical ethics and controlled trials. BMJ 1963; 1: 1043-1049.

${ }^{2}$ Fried C. Medical experimentation: personal integrity and social policy. New York, NY: American Elsevier Publishing, 1974.

${ }^{3}$ Freedman B. Equipoise and the ethics of clinical research. N Engl J Med 1987; 317: 141-145.

${ }^{4}$ Lyden P, Meyer B, Hemmen T, Rapp K. An ethical hierarchy for decision making during medical emergencies. Ann Neurol 2010; 67: 434-440.

${ }^{5}$ Braunholtz DA, Edwards SJ, Lilford RJ. Are randomized clinical trials good for us (in the short term)? Evidence for a "trial effect." J Clin Epidemiol 2001; 54: 217-224.
} 
others) that found no effect. ${ }^{6}$ Second, they allude to other features of clinical trials that may be beneficial to enrolled patients, such as more intensive monitoring and risk factor management, or earlier access to new interventions. This represents a rather selective view, as they give no consideration to other features of clinical trials that limit the quality of care available to research participants for the sake of producing generalizable data. These include not only randomization but also washout periods, placebo controls, restricted flexibility in the dosing of study drugs, restrictions on the use of concomitant treatments, invasive data-gathering interventions such as blood draws and lumbar punctures, sham surgeries and other interventions performed to preserve blinding, and imaging procedures performed not for the sake of guiding treatment but instead for evaluating efficacy. (It should also be noted that some of the beneficial features they cite are not intrinsic to the clinical research setting - if patients do in fact receive more effective monitoring and risk factor management in clinical trials than in routine care, this may not suggest that more patients should be enrolled in clinical trials, but instead that our models for the delivery of routine care must be improved.)

Lyden and colleagues begin their article with the arresting anecdote of a young patient with a devastating right middle cerebral artery occlusion who failed to respond to standard treatment with recombinant tissue plasminogen activator, was enrolled in an investigational protocol, and was then randomly assigned to the active treatment arm, underwent embolectomy, and had a good outcome. According to their arguments, it would have been unethical, for instance, for a physician convinced of the utility of embolectomy to empirically proceed to embolectomy after the patient failed to respond to standard treatment, without first subjecting the patient to randomization; furthermore, they base this in part on the claim that trial enrollment and randomization are best for the individual patient. This last claim strikes me as unsupported. First, a better case for comparison against this empiric treatment would be 1 of the equally numerous patients in the study who fail to respond to standard treatment and then are randomized to the control arm (standard care without embolectomy), as this is the circumstance in which trial enrollment and randomization make a clear difference. Second, there is no reason in this case to suspect that the adjunctive care available to the empirically treated patient will be inferior to the adjunctive care available in the study; and because this is an acute intervention, access to future improvements would be irrelevant. It is true that, as the authors note, empiric treatment would expose the patient to risks and would provide no proven benefit-but, of course, so would enrollment in a clinical trial, as clinical trials exist in order to expose subjects to unproven treatments and measure their effects.

I make these points to emphasize what clinical researchers and bioethicists have long recognized: that there are deep moral conflicts at the heart of clinical research, between the clinical aim of providing care to the individual patient and the scientific aim of producing generalizable data to guide the care of future patients.

The Therapeutic Misconception

\footnotetext{
${ }^{6}$ Vist GE, Bryant D, Somerville L, et al. Outcomes of patients who participate in randomized controlled trials compared to similar patients receiving similar interventions who do not participate. Cochrane Database Syst Rev 2008; (3): MR000009.
} 
In 1982, Paul Appelbaum and colleagues published a troubling and groundbreaking study documenting what they termed the "therapeutic misconception" in clinical research. ${ }^{7,8}$ They interviewed a group of psychiatric patients immediately after they had given informed consent to participate in a randomized, double-blinded, and placebo-controlled clinical trial; the consent process was described as "nearly ideal," including discussions about the study's design with the principal investigator that in some cases lasted for several hours. Nonetheless, a majority of subjects did not understand that treatment assignment would be random, and a substantial minority expressed the belief that treatment assignment would be based on an individualized assessment of their clinical needs. A third of the subjects did not recognize the role of blinding, with some expressing the belief that their treating physician would decide on the treatment best for them, and almost none of the subjects recognized that the choice and dosing of their medications would be limited by the study protocol. Subsequent studies by these authors and others have found similarly prevalent misconceptions among participants in clinical research with nonpsychiatric indications.

Although there is disagreement about the scope of the therapeutic misconception (with some authors using the term in unhelpfully broad ways), ${ }^{10} \mathrm{I}$ believe that at the core of this misconception is a failure to recognize ways in which the scientific prerogatives of clinical research limit the provision of individualized care to trial participants. (It is not a misconception, in my view, merely for a patient to enter a clinical trial with therapeutic intent, or even for a patient to judge that trial enrollment in his particular case offers, on balance, a better bet than routine clinical care.) Therapeutic misconceptions on the part of clinical trial participants are problematic because they undermine informed consent-for instance, if a patient in a clinical trial continues to believe, despite having been informed to the contrary, that she will be assigned to a treatment arm based on an individualized assessment of her clinical needs and not by a random draw, then it is doubtful that she really understands what she has consented to.

The therapeutic misconception is not necessarily limited to patients, but may influence clinical investigators as well. Presumably this is not because clinical investigators fail to understand basic features of trial design such as randomization, blinding, or restricted flexibility in dosing within a study protocol. However, there is a danger that the dual role of scientist and clinician, with competing commitments to important societal and patient-centered aims, may invite a form of cognitive dissonance that leads investigators to overestimate the generic benefits of clinical trial enrollment, or to overlook features of their study designs that limit the provision of individualized care to study participants. Therapeutic misconceptions on the part of clinical investigators also undermine informed consent, as they bias investigators' discussions with patients regarding the risks and benefits of trial enrollment. In addition, clinical investigators have a responsibility to choose research questions and design studies so as to limit research-related risks to participants and manage conflicts between scientific and clinical aims. To do so effectively, they must acknowledge that such conflicts exist.

\footnotetext{
${ }^{7}$ Appelbaum PS, Roth LH, Lidz C. The therapeutic misconception: informed consent in psychiatric research. Int J Law Psychiatry 1982; 5: 319-329.

8 Appelbaum PS, Roth LH, Lidz CW, et al. False hopes and best data: consent to research and the therapeutic misconception. Hastings Cent Rep 1987; 17: 20-24.

${ }^{9}$ Appelbaum PS, Lidz CW, Grisso T. Therapeutic misconception in clinical research: frequency and risk factors. IRB 2004; 26: 1-8.

${ }^{10}$ Kimmelman J. The therapeutic misconception at 25: treatment, research, and confusion.Hastings Cent Rep 2007; 37 : $36-42$.
} 


\section{Proposals for Managing Moral Conflict}

Several prominent bioethicists have argued that the therapeutic misconception is so pervasive that the only appropriate response is to promulgate new ethical standards for clinical research that are divorced from the ethics of clinical care-thus, they claim, clinical investigators should view themselves purely as scientists and not also as doctors, and trial participants should view themselves purely as research subjects and not also as patients. ${ }^{11,12}$ Although this radical move may appear to reduce ambiguity, it would carry great practical and conceptual costs. ${ }^{13}$ Instead, we should first recognize that clinical research is only 1 of several domains in which patient-centered clinical aims may exist in tension with other legitimate societal aims -2 others are medical education and efforts at cost control. ${ }^{14}$ Thus, the moral conflicts discussed here are not unique to clinical researchers, but instead reflect a more general problem: how to strike an appropriate balance between the interests of present and future patients.

In obtaining informed consent for trial enrollment, investigators should take pains to emphasize to prospective participants that enrollment in a clinical trial differs in important ways from ordinary clinical care; in particular, whereas ordinary clinical care is guided by the clinical aim of benefiting the individual patient, care within the context of a clinical trial is guided by the dual aims of benefiting the individual patient and producing generalizable data to guide the care of future patients. Thus, although clinical trial enrollment may have benefits for the individual patient relative to ordinary clinical care (such as the prospect of access to novel treatments), it also involves risks and tradeoffs not encountered in ordinary clinical care. For this reason, the aims of participants and investigators will be best aligned in cases where patients have altruistic as well as personal reasons for enrolling.

Furthermore, in the design of clinical trials, investigators should consider practical study designs that increase the latitude available to optimize the care of study participants while preserving the validity and generalizability of the data collected. ${ }^{15}$ Such efforts may have scientific as well as clinical benefits. First, the findings of such trials are often more generalizable, as they better reflect clinical decision making in the context of routine care. Second, the use of more flexible study protocols may reduce the temptation, reported by many investigators, to deviate from protocols in ways that may benefit the patient but ultimately jeopardize the validity of the data collected. ${ }^{16}$ Such temptations illustrate that a failure to recognize a tension between clinical and scientific aims may lead to both aims being undermined. Here again, it is preferable that the tensions between clinical and scientific aims are acknowledged by study participants and investigators at the outset.

Given these complexities, a physician's decision to recommend enrollment in a clinical trial must depend on an individualized assessment of the patient, the clinical question, and the specific

\footnotetext{
${ }^{11}$ Miller FG, Brody H. A critique of clinical equipoise: therapeutic misconception in the ethics of clinical trials. Hastings Cent Rep 2003; 33: 19-28.

${ }^{12}$ Miller FG, Rosenstein DL. The therapeutic orientation to clinical trials. N Engl J Med 2003; 348: $1383-1386$.

${ }^{13}$ Chiong W. The real problem with equipoise. Am J Bioeth 2006; 6: 37-47.

${ }^{14}$ Chiong W. Justifying patient risks associated with medical education. JAMA 2007; 298: 1046-1048.

15 Tunis SR, Stryer DB, Clancy CM. Practical clinical trials: increasing the value of clinical research for decision making in clinical and healthy policy. JAMA 2003; 290: 1624-1632.

${ }^{16} \mathrm{Lidz}$ CW, Appelbaum PS, Joffe S, Albert K, Rosenbaum J, Simon L. Competing commitments in clinical trials. IRB 2009; 31: 1-6.
} 
design of the clinical trial under consideration. There may be many circumstances in which clinical trial enrollment offers a better bet for an individual patient than routine clinical care; however, it would be unreasonable to assume that this is so in all cases. There may be other circumstances in which trial enrollment would disadvantage the patient in minor ways, but the scientific question is of urgent interest to many future patients, in which case it may be reasonable to ask the patient to assume these research-related tradeoffs for the sake of benefiting others-especially for patients who are, at least in part, altruistically motivated. Considering these many different possibilities, it seems very unlikely that a blanket hierarchy, such as that proposed by Lyden and colleagues, will be a useful tool in helping physicians and clinical investigators to manage the moral conflicts that arise in clinical trial enrollment. ${ }^{17}$

\footnotetext{
${ }^{17}$ Merritt M. Moral conflict in clinical trials. Ethics 2005; 115: 306-330.
} 